A Complete Conceptual Model

\title{
A Complete Conceptual Model for the Integrated Management of the Transportation Work
}

\author{
Yannis Tyrinopoulos, Aristotle University of Thessaloniki \\ Hellenic Institute of Transport
}

\begin{abstract}
Public transport faces a challenging period in which relevant operators invest in telematics systems in order to upgrade their internal operations and improve the services provided. Moreover, the public transport system has become quite dynamic during recent years, in the sense that the performance, effectiveness, and quality of its processes depend, to a large extent, on the factors affecting the vehicle's daily transportation work. Under the Ph.D. program at the Aristotle University of Thessaloniki, Telematics Applications in Public Transport, the lack of a complete management information model, that will effectively integrate traditionally static and dynamic public transport operations was identified. This article provides an overview of the management information model MIDAS-the result of a six-year research and development effort, aimed at fulfilling this gap. The article puts particular emphasis on the methodology applied and the techniques employed for development of the model. MIDAS contains three types of interrelated models: data model, functional model, and dynamic model. These models are the basis for the design and development of information systems for the planning and management of public transport operations.
\end{abstract}




\section{Introduction}

This article provides an overview of the integrated management information model MIDAS, developed in the context of the Telematics Applications in Public Transport Ph.D. program at Aristotle University of Thessaloniki. The particular needs served by the model are explored, with emphasis on the methodology adopted and techniques employed for its development.

The ultimate goals of telematics applications in the public transport sector are to improve the services offered and to optimize them overall in economic and environmental terms by means of intermodal and information communication mechanisms. These goals can be specified even further through the provision of high-quality information covering multiple systems. Thus, more efficient use of existing transport infrastructures and smoother transport flows enhance efficiency and safety, as well as reduce energy consumptions and accidents. The Association of German Transport Undertakings (VDV), which has a long presence in public sector telematics and published "Telematics in Public Transport in Germany" (2001), notes that telematics services are based on fast data transfer making use of advanced telecommunication networks and the processing of the exchanged data through hardware and software.

Recent trends in the public transport sector, as well as in the wider transport sector, imply that relevant operators adopt integrated information technology (IT) solutions to unify the technological environment. Giannopoulos and Tyrinopoulos (1999) demonstrated that, especially in public transport, this integration is even more compulsory, because operators often invest in different software applications for specific corresponding functional domains. Giannopoulos and Tyrinopoulos noted that operators also handle an extensive volume of data generated by a variety of sources. Therefore, the interoperability and interconnectivity of these software applications and data sources are significant goals to achieve in order to maximize the benefits for public transport operators.

\section{The Need}

\section{Lack of Operability Element and Its Consequences}

Information is an asset for all modern public transport operators that want to upgrade their computerization infrastructure and improve services provided to customers (passengers). Information has to be accurate, comprehensive, and provided in a timely manner. However, efficient information flow, accuracy, reliability, 
and comprehensiveness are directly associated with effective interoperability between various applications handling this information. Achieving interoperability means faster information communication, timely information provision, and an effective decision-making process.

A basic conclusion reached in the context of the European Union TITAN (TRANSMODEL-based Integration of Transport Applications and Normalization) research project and a concern expressed by many European public sector operators is that a significant volume of data is still communicated between different software applications in manual forms. Other important conclusions drawn are:

- The same data is generated by different sources (e.g., manual measurement of run times in the field and data collection through an automatic vehicle monitoring system).

- The same data is imported more than once in different software applications or data stores.

- There is incompatibility between data used by different software applications (e.g., different distances between the same bus stops in the scheduling system, in the automatic vehicle monitoring system, and in the GIS).

Figure 1 shows the interaction between various indicative key operations of a public sector operator in the form of basic information flows between them. It attests that interoperability between different functional domains is an important need for public transport operators (mainly medium and large sized). Two approaches encourage this interoperability: (1) multiple interfaces between different applications; and (2) a centralized approach through data sharing and handling.

Although multiple interfaces between different applications have, in practice, been proved inefficient, this approach has been unable to provide sufficient data sharing and communication. It is prone to errors and delays in information availability. In addition, this approach offers limited information reliability and substantially increases applications operation and maintenance costs. Tyrinopoulos and Patrikalakis (2000) thoroughly explored the main problems that currently exist due to the lack of an effective mechanism of IT applications integration in the public sector, including:

- incompatibility between applications

- insufficient information flow

- multiple storage of common datasets 


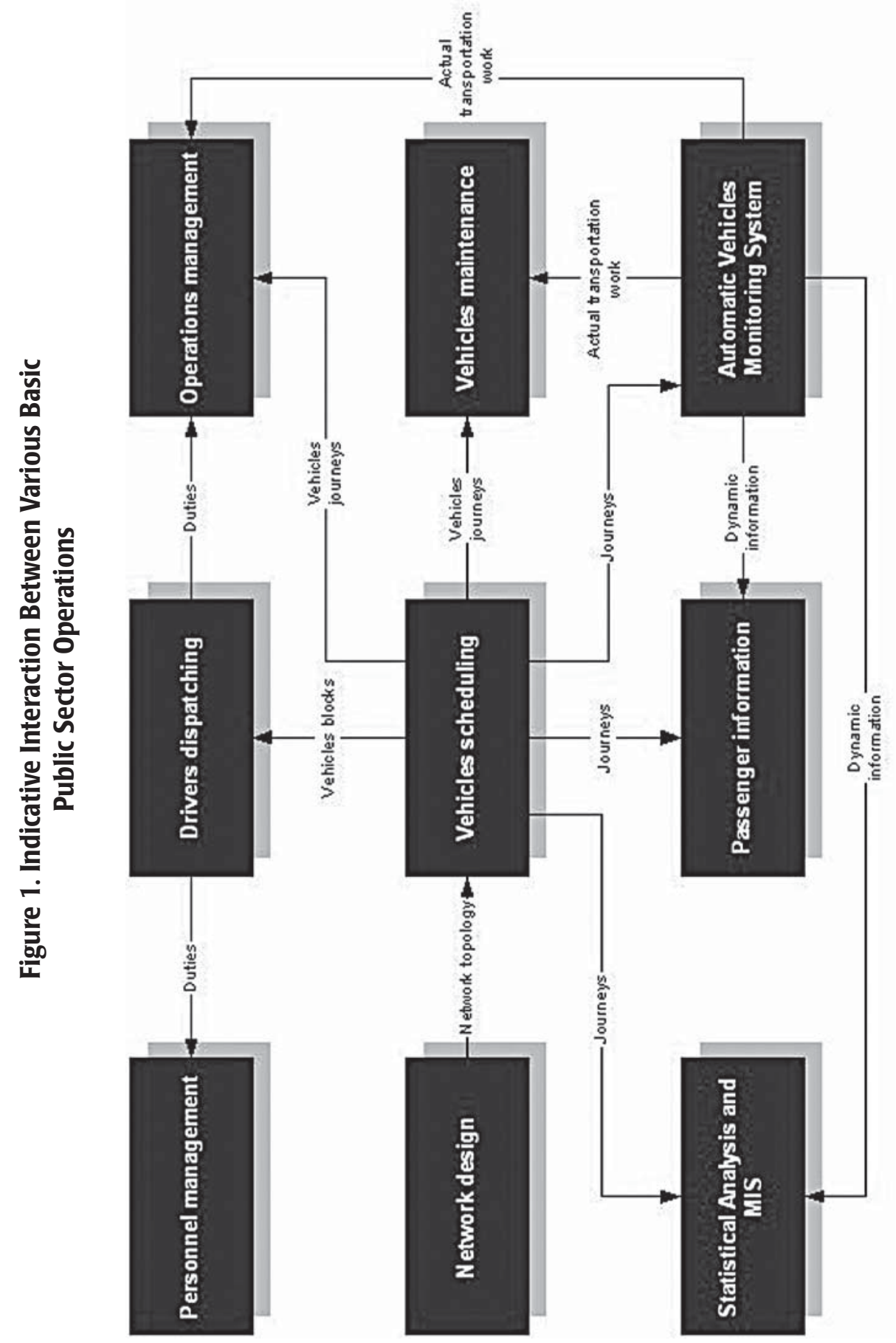


- delays in information availability

- high operation and maintenance costs of the applications

The centralized approach includes unification of the technological environment of a public transport operator, integrating the various IT applications. In particular, the public transport operator stores all information in a central database, which is accessible to all related applications. This approach is depicted in Figure 2.

The integrated computerization infrastructures based on centralized data sharing and handling are facilitated by the existence of a complete management information model. Such a model simulates the integration and data communication processes between different applications, and generates an uninterrupted and robust chain of information systems (see Figure 2).

\section{Current Situation in Public Transport Modeling}

Modeling in IT is neither a new nor even recent activity. Its first most significance steps can be found during the mid-1980s. Up to now, IT experts acknowledge that the existence of a complete information model consists of a foremost prerequisite for the development of an efficient, cost-effective, and robust information system.

A thorough review of management information models available in public transport from 1988-2000 was undertaken. The review contained both models dedicated to public transport operations and models covering the wider transport sector, which also include public transport operations. The most important and well-known models identified are:

- Models Dedicated to Public Transport Operations: ÖPNV Data Model (Association of German Transport Undertakings 1999), TRANSMODEL (Bert, Bouree, and Staub 1996), TCIP, ATCO-CIF, TransXChange, and VESCOS.

- Models Covering the Wider Transport Sector: DATEX, ITS Architectures [eight total: United States, Europe (KAREN), Canada, Japan, Australia, Korea, China and Taiwan], and ITS Standards (developed by ISO, CEN, NTCIP, etc.).

Analysis of the models took place at two levels: (1) according to their modeling direction taken (functional models, data models, dynamic models, and interfaces); and (2) according to the public transport processes covered.

With regard to the direction taken by the models, most modeling efforts (52\%; see Figure 3) put emphasis on the data element of the information systems, deriving corresponding data models. The main reason, according to their developers, is 


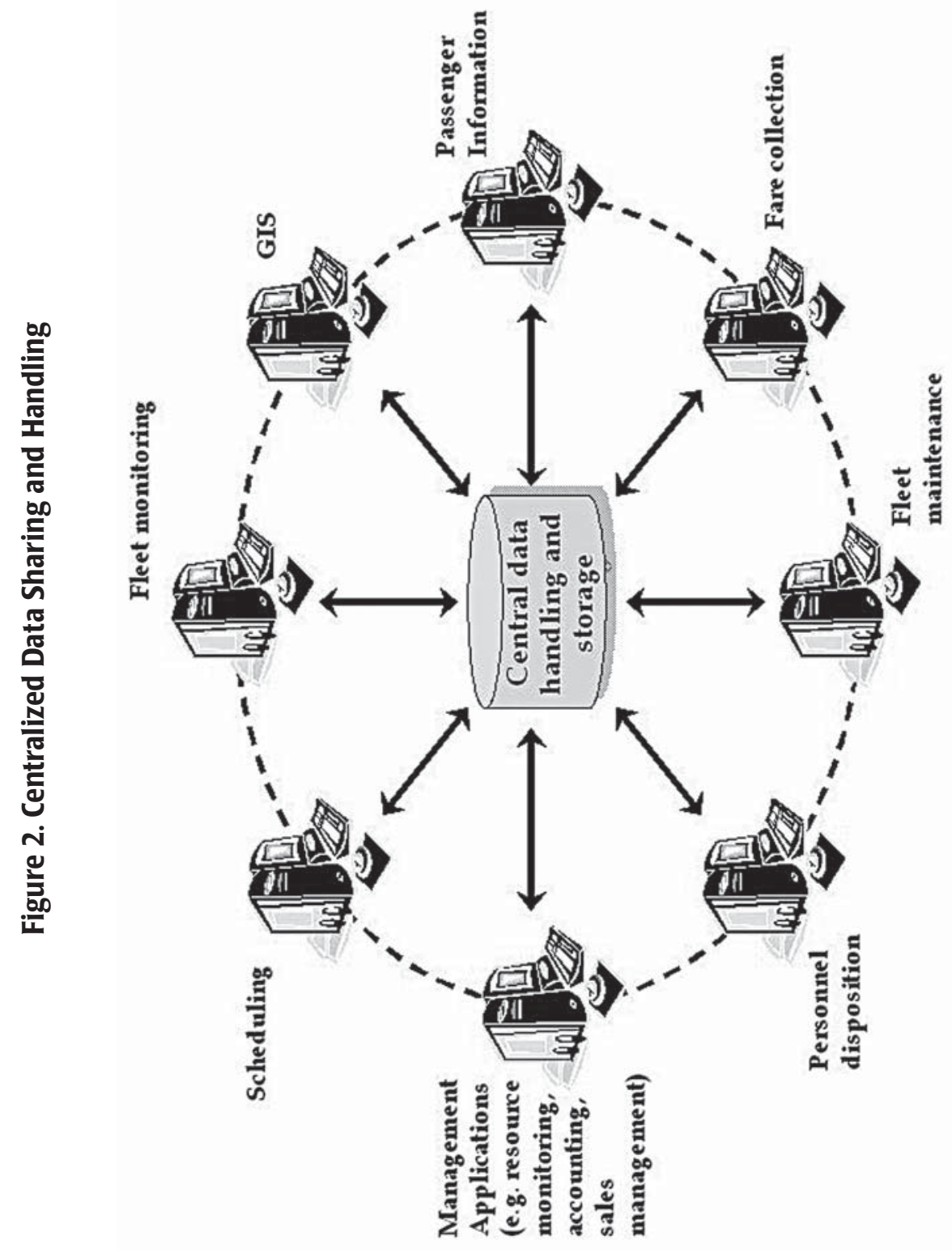


that the information needs of a public transport operator remain stable over time, while the operations have a more dynamic character and thus are not necessary to be modeled. Furthermore, none of the available models is complete, in the sense of covering all three main model types.

\section{Figure 3. Types of Models Available in the Public Transport Business}

With regard to the business processes, most models examine the public transport

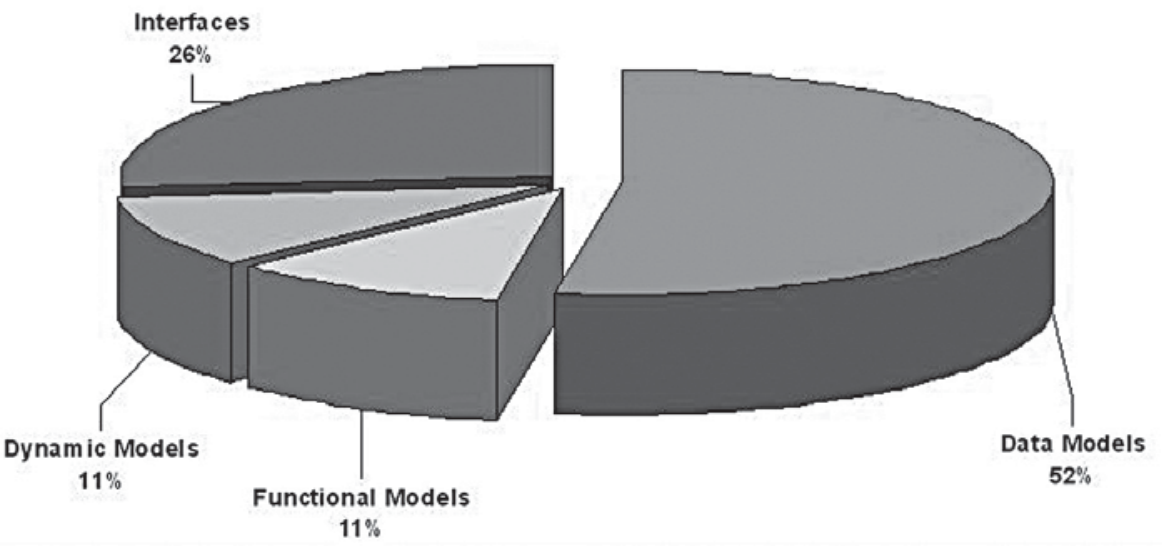

system as a static system. In particular, most models focus on the static processes of a public transport operator. Static processes are those functions that remain unchangeable for a long period of time (e.g., network design, scheduling, personnel disposition, and fare collection). Critical processes of dynamic nature are covered only peripherally and by a couple of models. Dynamic processes are those functions that are constantly modified over time, when an element of the process changes its value or characteristic. Such dynamic processes are Automatic Vehicle Location ( $A V L$ ) and real-time passenger information.

Current modeling initiatives in public transport place significant emphasis on the static elements of the operator, and center their attention on the scheduling phase of the transportation work. Respective analysis highlighted the lack of and need for a complete and integrated management information model that examines both static and dynamic public transport processes. 


\section{Introduction to MIDAS}

Responding to the needs identified above, the integrated management information model, MIDAS, was developed in the context of a Ph.D. program at the Aristotle University of Thessaloniki, Greece. The rationale behind the development of MIDAS was that such a modeling initiative should examine public transport as an integrated and dynamic system. In effect, the backbone of the model is the triptych: data, functions (business processes), and time. Based on this triptych, corresponding and interrelated types of models were developed: data, functional, and dynamic.

Two basic innovative features that differentiate MIDAS from other models available are:

- MIDAS has put significant emphasis on the dynamic character of the public transport system by modeling business processes, such as fleet monitoring, interchange control and protection, support of transportation operations, and real-time information provision to passengers.

- MIDAS has modeled all three elements of an information system (i.e., data, functions, and time) through the development of data models, functional models, and dynamic models, respectively.

Apart from the above, MIDAS is an open architecture effort. Its flexible design will enable it to handle future needs. It is equipped with friendly and specialized modeling techniques so that it can be understood and used by both public transport operators and software providers.

The development of MIDAS was focused on the strategic business processes of a public transport operator. In particular, emphasis was placed on scheduling and (real-time) management of vehicles and drivers and related operations (network topology, passenger information, etc.). Additionally, the model includes essential components of real-time management of transit operations, such as control and protection of interchanges and dispatching. An important element of the model is that it effectively integrates traditionally static and dynamic public transport operations.

Following a top-down decomposition analysis, the overall business area under consideration has been broken down into functional areas, functions, and processes. Figure 4 depicts the business processes modeled in MIDAS. 


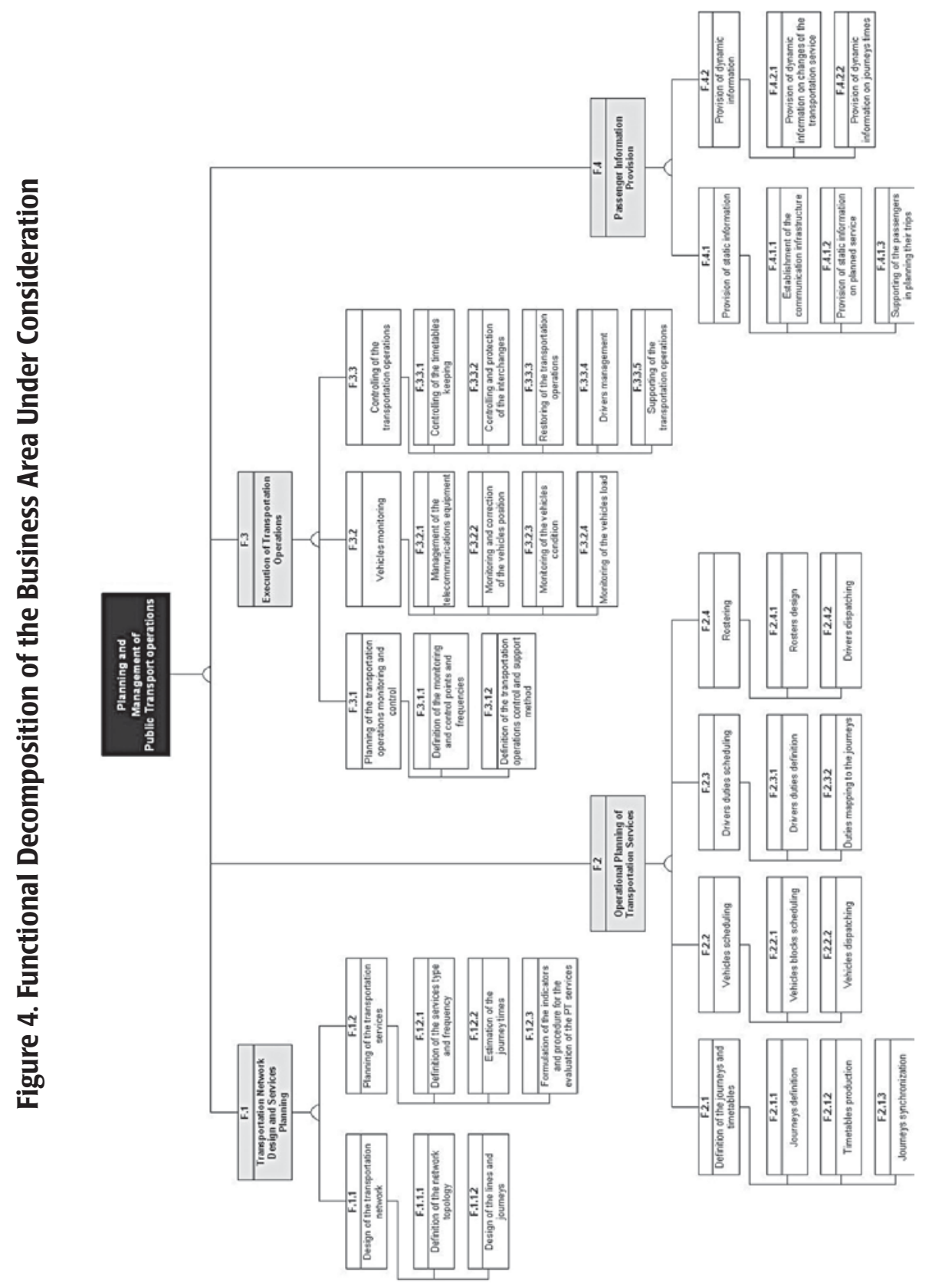




\section{Methodology}

Many methodologies have been developed for the analysis, design, and implementation of information systems. Some of the most well known are: Structured Systems Analysis and Design Method (SSADM), Information Engineering (IE), Yourdon Systems Method (YSM), and Jackson Systems Development (JSD). Avison and Fitzgerald (1995) explain that the choice for the adoption of the most appropriate methodology is subject to several criteria, such as the scope, nature, and functionality of the system under implementation.

MIDAS was based on the adoption of a quite popular and well-established methodology for the analysis and design of information systems-IE. James Martin, one of the main architects of this methodology, describes IE as "the application of an interlocking set of formal techniques for the planning, analysis, design, and construction of information systems on an enterprise-wide basis or across major sections of the enterprise" (1989). Figure 5 presents the classical representation (pyramid) of the IE methodology.

\section{Figure 5. IE Methodology Pyramid}

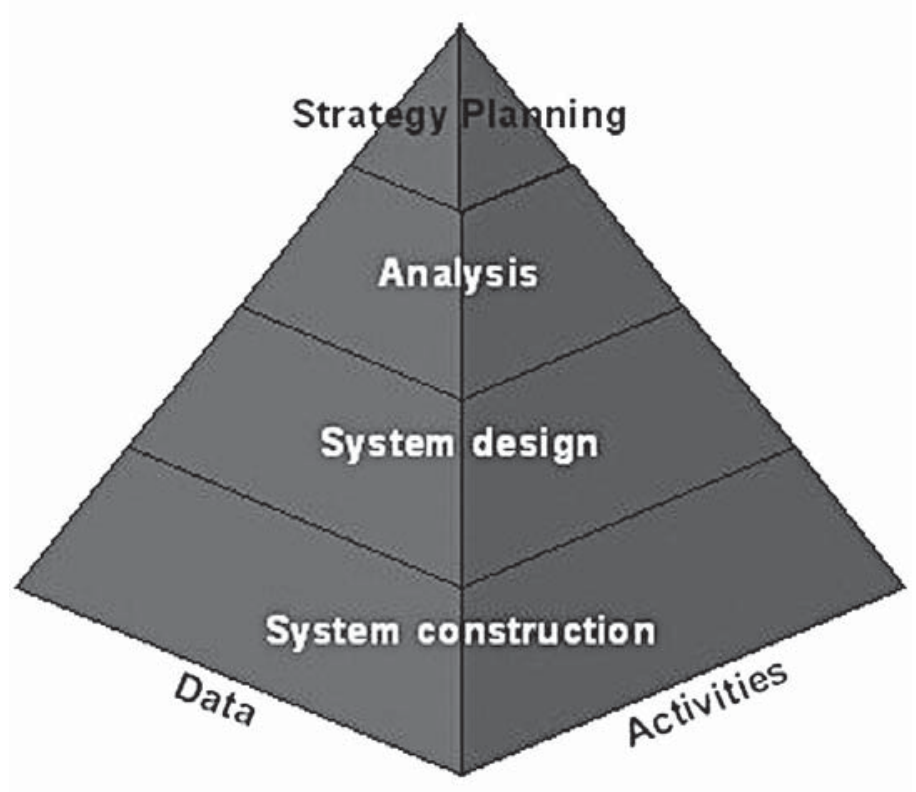


The left-hand side of the IE pyramid relates to data handled by the system; the right-hand side relates to activities/processes of the system. The four levels of tasks in the implementation of IE are:

- Strategy Planning. This level addresses technology and how it can be used to create new opportunities or competitive advantages. Strategy planning creates a high-level overview of the functions and information needs of the enterprise.

- Analysis. This level investigates processes needed to run a selected business area, how these processes interrelate, and what data is needed. It delivers data models and process models for the specific business area.

- System Design. This level examines how selected processes in the business area are implemented in procedures and how these procedures work. The procedures implementation (i.e., system) is designed with the help of automated tools.

- System Construction. At this level, the system is constructed with the help of automated tools, such as code generators and programming languages, and coupled with system design tools.

This specific methodology was selected because it addressed the needs of MIDAS and followed a business approach for the analysis and design of information systems. Furthermore, IE allows a structured and hierarchical analysis of the business areas under consideration, uses simple but comprehensive diagrammatic techniques, and involves the users in all its stages.

\section{Modeling Techniques}

Specialized modeling/diagrammatic techniques were employed for the design and presentation of the integrated model. These are:

- Decomposition diagrams for the decomposition of a functional area into lower-level functions and processes.

- Entity-relationship diagrams for modeling the database schema of MIDAS.

- Data flow diagrams for modeling the business processes and their interrelations, in terms of data flows.

- Sequence diagrams for incorporating the dimension of time and, in particular, the effect of time to the business processes.

- Entity life histories for analyzing the effects of time to the data elements. 
Journal of Public Transportation, Vol. 7, No. 4, 2004

- Processes-entities matrix for mapping entities of the data model to business processes of the functional model and also for validation purposes.

- Data dictionary for providing comprehensive storage of all elements (data and functions) used by MIDAS.

More information for most of the above modeling techniques can be found in Edward Yourdon's best-selling book, Modern Structured Analysis (1989).

\section{MIDAS Structure}

\section{Data Models}

This type of modeling identifies, analyzes, and models the information needs of a business process. The end result of this procedure is a data model that contains the description, structure, and organization of all data necessary for the execution of this business process.

In MIDAS, distinctive as well as interrelated data models were developed for 30 business processes at the lower level of the functional decomposition analysis (see Figure 4). The data model of each business process includes a description of the particular process from the point of view of the information needs of the process. This description highlights the entities that will store the data required for execution of the process. Moreover, the description is made to facilitate recognition of the attributes of the entities, as well as their relationships. Finally, the process description is accompanied by a synopsis of the entities required for its execution and ends up in one or more entity-relationship diagrams providing the complete data model for this process.

In developing MIDAS, all 30 data models underwent a normalization process (up to the third normal form) leading to the production of normalized data models. Normalization is a process of assigning attributes to relational tables to reduce data redundancy, eliminate data anomalies, and provide a robust data architecture for retrieving and modifying data.

Figure 6 demonstrates the entity-relationship diagram of a particular business process, which is also a subset of the integrated MIDAS data model. 


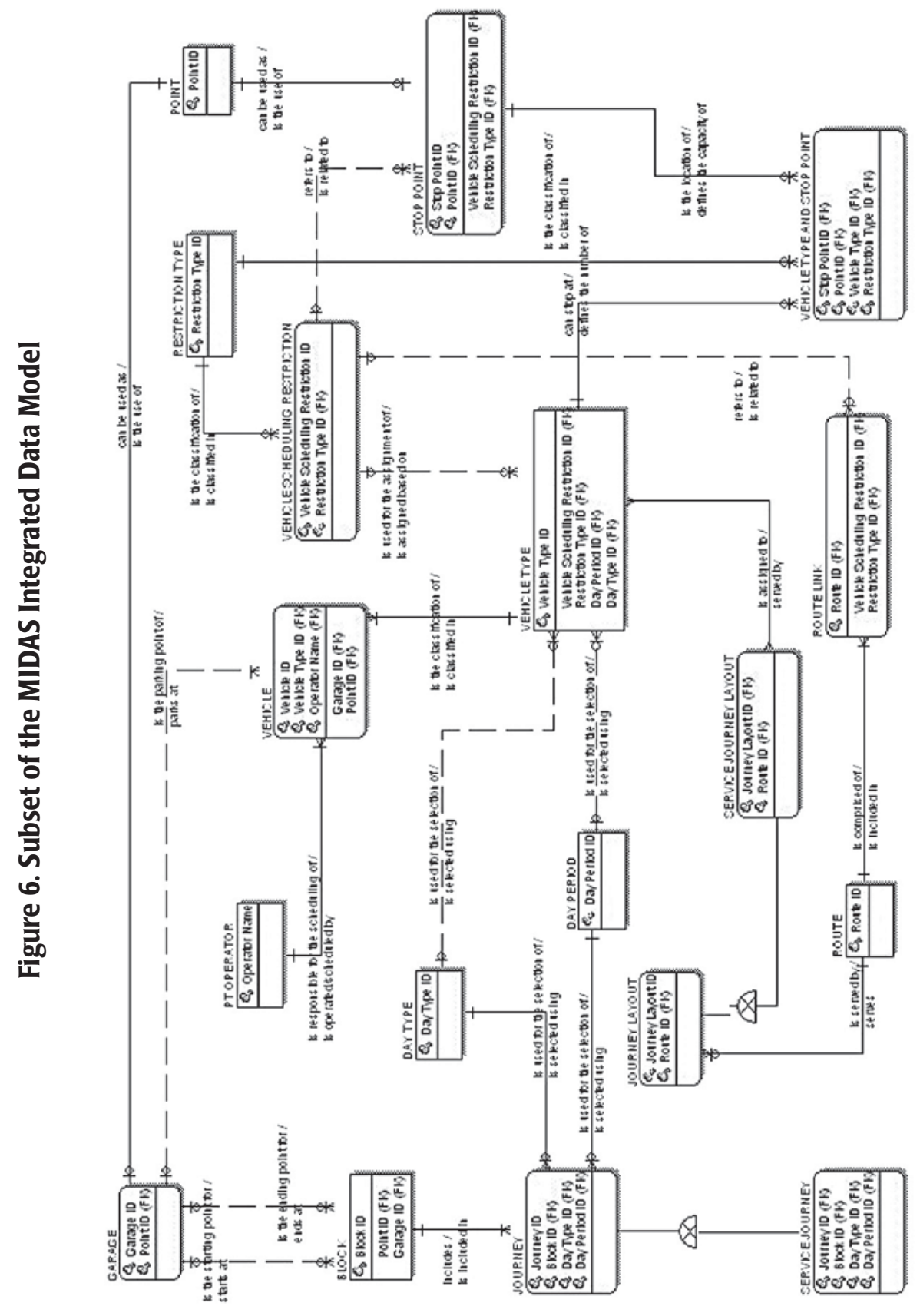


Figure 6 presents the entities, their key attributes, and the relationships between the entities of a specific business process. For example, in practice, the garage of a specific set of vehicles is associated with the blocks that the vehicles of this garage will occupy. In the data model, the relationship between the garage and the vehicle blocks has been modeled as follows (see the top left of Figure 6): the garage (entity GARAGE) is the starting point and the ending point of one or more blocks (entity BLOCK). The lines in the figure represent the relationships between the entities and are distinguished in "identifying" (solid lines) and "non-identifying" (dashed lines). Without entering into much technical discussion, in the identifying relationships one entity cannot exist without the associated one, while in the nonidentifying relationships, one entity just migrates its key data to the associated one.

\section{Functional Models}

In general, a functional model includes the functional specifications of an information system in the form of functions and information flows between them. In MIDAS, the business processes of a system that plans and manages vehicles and drivers were organized and classified in levels, while their interrelationships were modeled in the form of information flows between them.

The MIDAS functional models consist of a significant part of the integrated management information model MIDAS, since they substantially contribute to its understanding, comprehensiveness, and applicability. In addition, the MIDAS functional models provide the corresponding data models with the necessary business and functional background, without which the latter cannot exist.

The decomposition diagrams and data flow diagrams are the modeling techniques employed for the development of the MIDAS functional models. The first technique, following a top-down approach, decomposes the business area under consideration in functions at lower levels, while the second allows the abovementioned information system to form as a network of functional processes, connected to one another by canals and stores of data (see Figure 7).

\section{Dynamic Models}

The dynamic model completes the management information model. This model type adds the dimension of time to the integrated model. Particularly, an integrated description of an information system demands the development of a dynamic model, which demonstrates how various components of the system behave in specific time frames and the effect of one on another. 


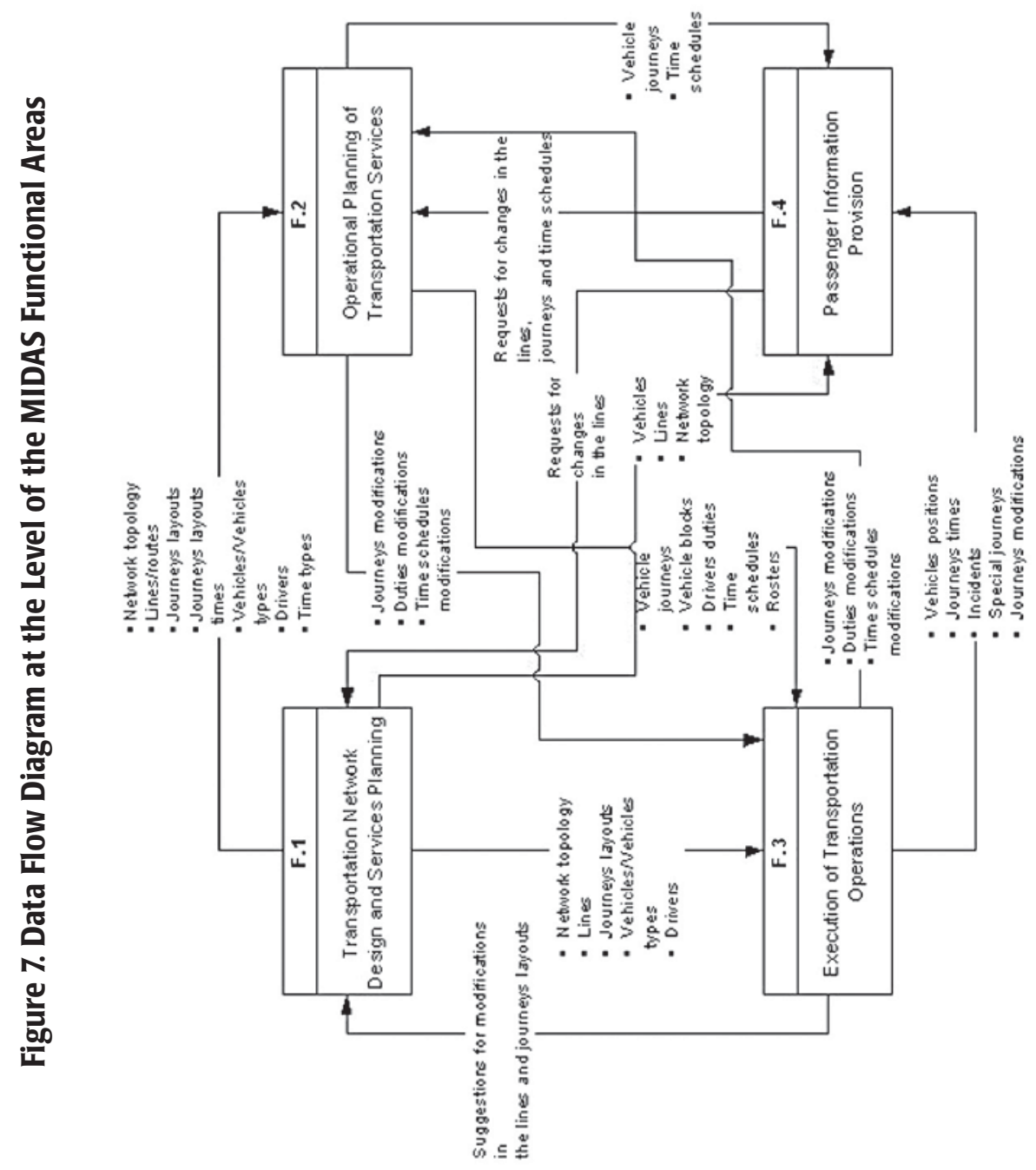




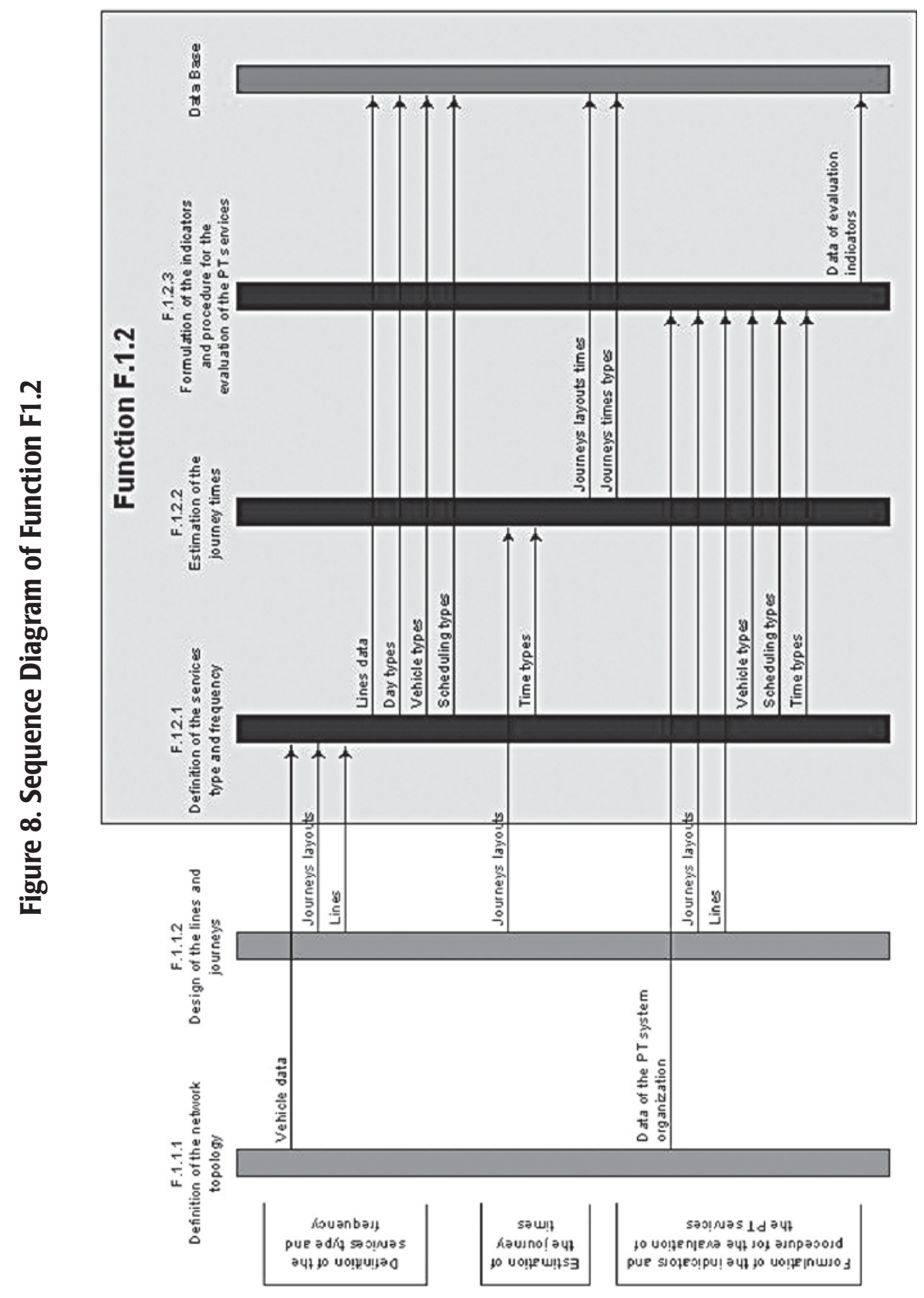


The dynamic character of the public transport system imposes the development of a set of dynamic models, especially since the business area under consideration covers processes that extensively incorporate the dimension of time. This dimension affects not only the processes of an information system, but also the data that these processes handle. To model these two different aspects, two corresponding modeling techniques were employed.

- Sequence diagrams focus on the interaction between functions and the time sequence of the exchange of information flows between functions (see Figure 8).

- Entity life histories examine the effects that events happening to an information system have on entities (data stores). The power of this technique is quite apparent when an entity changes state frequently (see Figure 9).

\section{Figure 9. Entity Life History (ELH) Diagram of the Entity "Vehicle"}

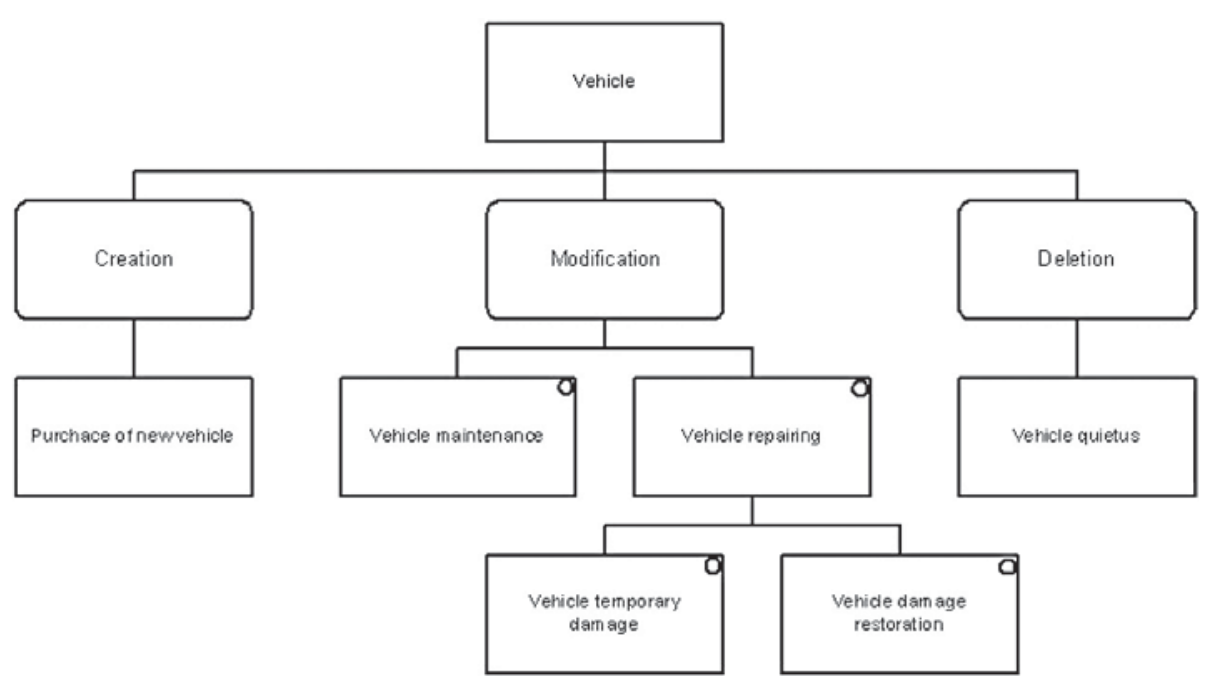


All three MIDAS types of models presented above are accompanied by a data dictionary. The MIDAS data dictionary provides an organized list of all data elements (entities and information flows) with concise, precise, and rigorous definitions, so that both users and systems analysts will have a common understanding of all inputs, outputs, and components of stores.

\section{Driving Forces of the Model}

MIDAS follows the current trend in the European wider transport business for the development of integrated management information models. The EU, through research and development ( $R \& D$ ) programs and initiatives, places particular emphasis on the basic components of such models for the development of advanced IT solutions. MIDAS addresses the public transport domain by envisioning a reliable tool for stakeholder parties-public operators and software providers. Apart from the two basic innovative features of the model identified earlier, some of its most important characteristics that significantly contribute toward this direction are the following:

- The development of MIDAS was based on a well-established methodology, IE, which follows a process-oriented approach.

- MIDAS refers to vehicles and not specifically to busses, wagons, etc. Thus, it can be used by any public transport operator, regardless of the types of vehicles it operates. In effect, MIDAS also covers multimodal public transport.

- The functional model of MIDAS provides the integrated model with a necessary business dimension, incorporating business rules, needs, and constraints that govern the public transport system.

- MIDAS is an open architecture model that permits extensions toward the satisfaction of future operational and information needs.

- The modularity of the model allows adoption of specific models for the fulfillment of business needs of specific functional domains.

\section{Conclusions}

Public transport operators worldwide still experience significant problems in the scheduling and management of transportation services. Among the problems are high operation and maintenance costs of information systems provided by differ- 
ent software providers, information discontinuities, low levels of interoperability, multiple collections and archiving of the same data, and delays in information availability.

Integration is the key toward a more effective organization, scheduling, and operation of transportation activities. The integration process is a top priority for all modern public transport operators. Integration will help solve significant problems and discontinuities at operational, organizational, and informational levels.

Modern transportation theories and the constantly increasing needs of public transport operators require advanced computerization infrastructures, integrating systems of different functional domains, and incorporating both static and dynamic business processes. In recent years, emphasis has been placed on developing management information models that guide the implementation of advanced IT solutions. These models play a decisive role in the development of IT infrastructures, making feasible the integration concept.

MIDAS was developed to address the lack of a complete management information model that will effectively integrate traditionally static and dynamic public transport operations, as well as incorporate all three elements of an information system (functions, data, and time). The approach and specifications proposed by MIDAS provide significant gains for public transport operators, including:

- More effective collaboration between software providers (systems developers) and users (public transport operators).

- Facilitation of the specifications development process for public transport information systems.

- More efficient and cost-effective information systems development processes.

- Identification of business processes that can be improved or recognition of new ones.

- More efficient management of transportation services.

- Compatibility between information systems provided by different software providers.

- More rationale use of the existing IT infrastructure of a public transport operator.

MIDAS is a conceptual model and the aim of its developer is to implement it in the context of $R \& D$ projects and use it in the course of university lectures. The 
Journal of Public Transportation, Vol. 7, No. 4, 2004

latter has already been achieved at the Aristotle University of Thessaloniki, where modeling techniques, integrated model organization, and management of the transport work are being taught to post-graduate students. In regards to implementation, the active participation of the author in $\mathrm{R} \& \mathrm{D}$ programs will provide opportunities to implement the integrated model or part of it. 


\section{References}

Association of German Transport Undertakings (VDV). 1999. OPNV Data Model 5.0 for Public Transport. January.

Association of German Transport Undertakings (VDV). 2001. Telematics in Public Transport in Germany.

Avison, D. E., and G. Fitzgerald. 1995. Information Systems Development: Methodologies, Techniques and Tools, 2nd ed. McGraw-Hill.

Bert, Bruno, Kasia Bouree, and Lutz Staub. 1996. TRANSMODEL, Reference Data Model for Public Transport (European Prestandard). CEN TC278/WG3. Commission of the European Communities.

Giannopoulos, G. A., and Yannis Tyrinopoulos. 1999. Advanced telematics in public transport. Paper presented at the Conference on Urban Public Transport: Energy and Environment. Athens. October 18.

Martin, James. 1989. Information Engineering, Book I: Introduction. Prentice Hall PTR.

Tyrinopoulos, Yannis, and Yannis Patrikalakis. 2000. The integration of PT information systems through unified databases. Paper presented at the 13th National Conference of the Hellenic Operational Research Society, Transport and New Technologies. Piraeus University. November 30-December 2.

Yourdon, Edward. 1989. Modern Structured Analysis. Prentice-Hall International Inc.

\section{About the Author}

YANNIS TYRINOPOULUS (yannisty@kos.forthnet.gr) is a transportation engineer with a long and active presence in the application of advanced telematics systems to the transport sector and, in particular, public and freight transport. He obtained his Ph.D. at the Aristotle University of Thessaloniki. Dr. Tyrinopoulos is affiliated with the Hellenic Institute of Transport (HIT) at the Center for Research and Technology Hellas (CERTH). His main research interests are the analysis and design of advanced telematics applications in the transport sector. 